\title{
Possibility theory for multiobjective fuzzy random portfolio optimization
}

\author{
Mir Ehsan Hesam Sadati ${ }^{\mathbf{a}^{*}}$, Ali Doniavi ${ }^{\mathrm{a}}$ and Abbas Samadi ${ }^{\mathrm{b}}$
}

${ }^{a}$ Department of Industrial Engineering, Urmia University, Urmia, Iran

${ }^{b}$ Department of Industrial Engineering, Payame Noor University of Tabriz, Tabriz, Iran

\begin{tabular}{l}
\hline C H R O N I C L E \\
\hline Article history: \\
Received October 15, 2013 \\
Received in revised format \\
March 22014 \\
Accepted March 24, 2014 \\
Available online \\
April 72014 \\
\hline Keywords: \\
Multi-objective portfolio \\
optimization model \\
Possibility and Necessity-based \\
model \\
Fuzzy random variables
\end{tabular}
\begin{abstract}
A B S T R A C T
The problem of portfolio optimization is a standard problem in financial world and it has received tremendous attentions. Portfolio optimization plays essential role in determining portfolio strategies for investors. Portfolio optimization is intrinsically a discrete optimization problem whose decision criteria are in conflict and the proposed study of this paper considers a portfolio optimization problem involving fuzzy random variables. To solve the proposed model, we first present the possibility and necessity-based model to reformulate the fuzzy random portfolio selection model into linear programming models and using the resulted linear programs, a multi-objective problem is constructed. To solve the multi-objective problem we propose some methods to consider decision makers' optimistic and pessimistic views. A numerical example illustrates the whole idea on multiobjective fuzzy random portfolio optimization by possibility and necessity-based model.
\end{abstract}

(C) 2014 Growing Science Ltd. All rights reserved.

\section{Introduction}

Portfolio selection discusses the problem of how to choose appropriate combinations of assets among available ones so that the investment can bring the maximum investment return. In other words, portfolio selection helps managers concentrate between product and customer to invest resources in the best effective way. The investment return and risk always appear, simultaneously, in other words, portfolio optimization consists of the portfolio selection problem to determine the optimum approach of investment on a particular amount of money in a given set of securities or assets (Markowitz, 1952). Markowitz (1952) proposed mean-variance models for the portfolio selection problem and he formulated them mathematically in different ways such as minimizing variance for a given expected value, or maximizing expected value for a given variance. Since then, the mean-variance models have been well developed in both theory and algorithm (Crama \& Schyns 2003; Xia et al. 2000). The mean-variance models for the portfolio selection problem has been central to research activities of

* Corresponding author. Tel.: +98 9354326729

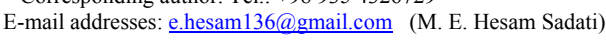

(C) 2014 Growing Science Ltd. All rights reserved.

doi: $10.5267 /$ j.dsl.2014.4.001 
this area and it has served as a basis for the development of modern financial theory for many years (Hao \& Liu 2008). In the term of development of modern financial theory, two examples are collected here: Yu \& Lee (2011) considered transaction cost, short selling and higher moments to achieve bigger flexibility in portfolio selection. They considered five portfolio rebalancing models, by considering transaction cost and by considering criteria such as including risk, return, short selling, skewness, and kurtosis to determine the important design criteria for a portfolio model. WoodsideOriakhi et al. (2013) considered the problem of rebalancing an existing financial portfolio, where transaction costs have to be paid if there is change in amount held of any asset and they modeled the problem as a mixed integer quadratic with an explicit on the amount that can be paid in transaction cost. Markowitz (1959) defined a semi-variance for as symmetric random returns since researchers pointed out that the asymmetric returns is not an appropriate method for measuring the risk. Many researchers studied the properties and computation problem about semi-variance (Grootveld \& Hallerbach, 1999; Markowitz, 1993) and developed the mean-semivariance models (Chow \& Denning, 1994).

Konno and Yamazaki (1991) introduced an advanced model in which a mean-absolute deviation (MAD) model and absolute deviation were utilized as a measure of risk. Since Markowitz (1952), portfolio theory has been greatly improved. The researches mainly focused on two aspects. On one hand, portfolio selection was investigated under stochastic environment, (see e.g. Harlow \& Rao, 1989. Plat (2009) proposed stochastic model for portfolio specific mortality experience, where the proposed stochastic process was applied to two insurance portfolios, and the impact on the Value at Risk for longevity risk was quantified. On the other hand, the portfolio problem was handled in fuzzy environment (e.g. Parra \& Terol, 2001). During the last few years, fuzzy portfolio selection have emerged and received a great deal of researchers (Sadjadi et al., 2011; Gharakhani \& Sadjadi, 2013; Dastkhan et al., 2013). Sadjadi et al. (2011) proposed a method in a form of fuzzy linear programming, which was capable of determining the amount of investment in different time cycles when determining of investment in different planning areas was primary concern and they considered return and borrowing/leading rate as fuzzy triangular numbers instead of crisp representations. Gharakhani and Sadjadi (2013) examined advanced optimization approach for portfolio problem introduced by Black and Litterman (1991) which proposed a new approach to estimate asset return to consider the shortcomings of Markowitz standard Mean-Variance optimization and they represented investor's view about future asset return by using fuzzy numbers. In addition, Dastkhan et al. (2013) dealt with application of three different operators of fuzzy mathematical programming in a meanabsolute deviation portfolio selection problem with real features of minimum transaction lots, fixed and proportional transaction cost, cardinality constraint and bounds on holding constraint.

Traditionally, returns of individual securities were considered as random variables under the assumptions that investors have enough historical data about security returns and the situation of asset markets in future can be correctly reflected by asset data in the past. However, these assumptions will be violated when new securities are listed in the market, or the real asset market is changed. To deal with this problem, researchers have made use of fuzzy set theory (Zadeh, 1965). In real world, the possibility distribution functions of security returns may be partially known. Then, fuzzy returns with random information appear. In fact, for an investor, the fuzziness and randomness of security returns are often mixed up with each other. Thus, the investor will be faced with fuzzy returns with random parameters. In such situations, we may employ fuzzy random theory (Liu, 2009) to deal with this twofold uncertainty of fuzziness and randomness. Random and fuzzy optimization models provide useful methods for investors to handle uncertainty. According to Sadjadi et al. (2012), portfolio problem becomes more complicated when the return of all risky assets are subject to uncertainty. They proposed a new portfolio modeling approach with uncertain data and it was also analyzed using different robust optimization techniques and Zhang et al. (2013) considered a multi-period portfolio selection problem imposed by return demand and risk control in a fuzzy investment environment, in which the returns of assets are characterized by fuzzy numbers. Liu 
et al. (2012) dealt with multi-period portfolio selection problems in fuzzy environment by considering some or all criteria, including return, transaction cost, risk and skewness of portfolio.

In reality, however, sometimes investors have to deal with the uncertainty of both randomness and fuzziness simultaneously. For example, security returns are usually regarded to be normally distributed random variables, but the expected values may be fuzzy. Hao and Liu (2009) developed two novel types of mean-variance models for portfolio selection problems, in which the security returns are assumed to be characterized by fuzzy random variables with known possibility and probability distributions. In their proposed models, expected return of a portfolio was as the investment return and the variance of the expected return of a portfolio was as the investment risk. In fact, from a practical viewpoint the fuzziness and randomness of security returns are often mixed up with each other, so fuzzy random variable can be a new useful approach to solve this kind of problem (Li \& Xu, 2009).

A fuzzy random variable was first introduced by Kwakernaak (1978), and its mathematical basis was constructed by Puri and Ralescu (1986). An overview of the developments of fuzzy random variables was found in the recent article of Gil et al. (2006). Shapiro (2009) implies that "Randomness models the stochastic variability of all possible outcomes of a situation, and fuzziness relates to the unsharp boundaries of the parameters of the model". In this paper, the assets return in portfolio selection problem are fuzzy random variables and we use the concept of possibility and necessity-based model to develop a solution method for the fuzzy random portfolio optimization problem and reformulate the portfolio optimization problem to the linear programming (Sadati \& Nematian, 2013). We construct multiobjective programming model by using these two types of linear programming to find the objective function value.

The rest of the paper is organized as follow: Section 2 includes basic concept on fuzzy and fuzzy random theory. In Section 3, the problem formulation is presented. In section 4, a numerical example is solved to show how our new method works. Finally, conclusion and future work will present in section 5 .

\section{Basic concepts}

A Fuzzy random variable was first introduced by Kwakernaak (1978), and its mathematical basis was constructed by Puri and Ralescu (1986). An overview of the developments of fuzzy random variables was found in the recent article of Gil et al. (2006). In general, fuzzy random variables can be defined in an $\mathrm{n}$ dimensional Euclidian space $\mathrm{R}^{\mathrm{n}}$. We present the definition of a fuzzy random variable in a single dimensional Euclidian space R.

\section{- Definition 1 (Sakawa 1993)}

Let $(\Omega, \mathrm{A}, \mathrm{P})$ be a probability space, where $\Omega$ is a sample space, $\mathrm{A}$ is a $\sigma$-field and $\mathrm{P}$ is a probability measure. Let $\mathrm{F}_{\mathrm{N}}$ be the set of all fuzzy numbers and $\mathrm{B}$ a Borel $\sigma$-field of $\mathrm{R}$. Then a map $\tilde{Z}: \Omega \rightarrow F$ is called a fuzzy random variable if it holds that

$\left\{(\omega, \tau) \in \Omega \times R \mid \tau \in \tilde{\bar{Z}}_{\alpha}(\omega)\right\} \in A \times B, \forall \alpha \in[0,1]$

where

$$
\tilde{Z}_{\alpha}(\omega)=\left[\tilde{\bar{Z}}_{\alpha}^{-}(\omega), \tilde{\bar{Z}}_{\alpha}^{+}(\omega)\right]=\left\{\tau \in R \mid \mu_{\bar{z}(\omega)}(\tau) \geq \alpha\right\}
$$

is an $\alpha$-level set of the fuzzy number $\tilde{Z}(\omega)$ for $\omega \in \Omega$.

Intuitively, fuzzy random variables are considered to be random variables whose realized values are not real values but fuzzy numbers or fuzzy sets. 


\section{- Definition 2}

LR fuzzy number $\tilde{Z}$ is defined by following membership function:

$$
\tilde{Z}(X)=\left\{\begin{array}{lll}
L\left(\frac{Z^{0}-x}{\beta}\right) & \text { if } & Z^{0}-\beta \leq x \leq Z^{0} \\
1 & \text { if } & Z^{0} \leq x \leq Z^{1} \\
R\left(\frac{x-Z^{1}}{\gamma}\right) & \text { if } & Z^{1} \leq x \leq Z^{1}+\gamma
\end{array}\right.
$$

where $\left[Z^{0}, Z^{1}\right]$ shows the peak of fuzzy number $\tilde{Z}$ and $\beta, \gamma$ represent the left and right spread respectively;

$L, R=[0,1] \rightarrow[0,1]$ with $L(0)=R(0)=1$ and $L(1)=R(1)=0$ are strictly decreasing, continuous functions. A possible representation of a LR fuzzy number is $\tilde{Z}=\left(Z^{0}, Z^{1}, \beta, \gamma\right)_{L R}$.

\section{Problem formulation}

Investors are rational and behave in a manner of maximizing their utility with a given level of income or money. In the following problem, called Fuzzy Random Optimal Portfolio selection problem, the return rate of assets are fuzzy random variables:

Problem 1

$$
\begin{aligned}
\max \tilde{Z}= & \sum_{j=1}^{n} \tilde{\bar{R}}_{j} x_{j} \\
\text { subject to } & \sum_{j=1}^{n} x_{j}=M_{0}, \\
& \sum_{j=1}^{n} \tilde{\bar{R}}_{j} x_{j} \geq \tilde{\bar{R}}_{0} \\
& 0 \leq x_{j} \leq U_{j} ; \quad j=1,2, \ldots, n .
\end{aligned}
$$

The parameters and variables are define as follow, for $\mathrm{j}=1,2, \ldots, \mathrm{n}$ :

$\tilde{\bar{R}}_{j}=\left(R_{j}^{0}, R_{j}^{1}, \beta_{j}, \gamma_{j}\right)_{L R}$ represent fuzzy random variables whose observed value for each $\omega \in \Omega$ is fuzzy number $\tilde{R}_{j}(\omega)=\left(R_{j}^{0}(\omega), R_{j}^{1}(\omega), \beta_{j}, \gamma_{j}\right)_{L R}$.

$\left(\bar{R}_{j}^{0}, \bar{R}_{j}^{1}\right)=\left(R_{j}^{0}+\overline{t R}_{j}^{2}, R_{j}^{1}+\overline{t R}_{j}^{2}\right)$ is a random vector in which $\bar{t}$ is a random variable with cumulative distribution function $T$.

$\mathrm{n}$ : the number of assets for possible investment

$\mathrm{M}_{0}$ : available total fund

$\tilde{\bar{R}}_{j}:$ the rate of return of assets $\mathrm{j}$ ( per period)

$\tilde{\bar{R}}_{0}:$ the return in dollars

$x_{j}$ : decision variables which represent the dollar amount of fund invested in asset $\mathrm{j}$

$U_{j}$ : the upper bound of investment in asset $\mathrm{j}$. 


\subsection{Possibility-based model}

By Zadeh's extension principle for objective function in problem 1, its membership function is given as follows for each $\omega \in \Omega$ :

$$
\mu_{\tilde{Z}(\omega)}(t)=\left\{\begin{array}{lll}
L\left(\frac{Z^{0}(\omega)-t}{\beta}\right) & \text { if } & t \leq Z^{0}(\omega) \\
1 & \text { if } & Z^{0}(\omega) \leq t \leq Z^{1}(\omega) \\
R\left(\frac{t-Z^{1}(\omega)}{\gamma}\right) & \text { if } & \text { otherwise }
\end{array}\right.
$$

where $\tilde{Z}(\omega)=\left(Z^{0}(\omega), Z^{1}(\omega), \beta, \gamma\right)_{L R}, Z^{0}(\omega)=\sum_{j=1}^{n} R_{j}^{0}(\omega) x_{j}$, and $Z^{1}(\omega)=\sum_{j=1}^{n} R_{j}^{1}(\omega) x_{j}$. The degree of possibility $\pi(\tilde{Z}(\omega) \geq f)$ under the possibility distribution $\mu_{\tilde{Z}(\omega)}(t)$ is given as follows:

$\pi(\tilde{Z}(\omega) \geq f)=\sup _{y_{1}, y_{2}}\left\{\min \left\{\mu_{\tilde{Z}(\omega)}\left(y_{1}\right), \mu_{f}\left(y_{2}\right)\right\} \mid y_{1} \geq y_{2}\right\} \geq \eta$.

The possibility degree of fuzzy constraint $\left(\sum_{j=1}^{n} \tilde{R}_{j}(\omega) x_{j} \geq \tilde{R}_{0}(\omega)\right)$ under the possibility distributions is defined as follows:

$\pi\left(\sum_{j=1}^{n} \tilde{R}_{j}(\omega) x_{j} \geq \tilde{R}_{0}(\omega)\right)=\sup _{y_{1}, y_{2}}\left\{\min \left\{\mu_{\sum_{j=1}^{n} \tilde{R}_{j}(\omega) x_{j}}\left(y_{1}\right), \mu_{\tilde{R}_{0}(\omega)}\left(y_{2}\right)\right\} \mid y_{1} \geq y_{2}\right\}$

We maximize the degree of possibility $\pi(\tilde{Z}(\omega) \geq f)$ and the degree of possibility $\pi\left(\sum_{j=1}^{n} \tilde{R}_{j}(\omega) x_{j} \geq \tilde{R}_{0}(\omega)\right)$, our portfolio selection model in Problem 1 comes by the following model:

\section{Problem 2}

$\max f$

subject to $\operatorname{Pr}\{\omega \mid \pi(\tilde{Z}(\omega) \geq f) \geq \eta\} \geq \lambda$,

$\sum_{j=1}^{n} x_{j}=M_{0}$

$\operatorname{Pr}\left\{\omega \mid \pi\left(\sum_{j=1}^{n} \tilde{R}_{j}(\omega) x_{j} \geq \tilde{R}_{0}(\omega)\right) \geq \eta\right\} \geq \lambda$,

$0 \leq x_{j} \leq U_{j} ; \quad j=1,2, \ldots, n$.

where $\lambda$ is a predetermined probability level and $\eta$ is a predetermined possibility level. A feasible solution of portfolio selection problem is called a possibility solution. In order to transform the above model to a linear programming model, we need to reformulate Eq. (12) and Eq. (14) in problem 2. Consider the following theorem:

Theorem 1: (Katagiri et al. 2008)

For any decision variable, it holds that: 


$$
\begin{aligned}
& 310 \\
& \left\{\begin{array}{l}
\text { 1) } \operatorname{Pr}\{\omega \mid \pi(\tilde{Z}(\omega) \geq f) \geq \eta\} \geq \lambda \\
\Leftrightarrow \sum_{j=1}^{n}\left(R_{j}^{1}+T^{*}(1-\lambda) R_{j}^{2}\right) x_{j}+R^{*}(\eta) \sum_{j=1}^{n} \gamma_{j} x_{j} \geq f \\
\text { 2) } \operatorname{Pr}\left\{\omega \mid \pi\left(\sum_{j=1}^{n} \tilde{R}_{j}(\omega) x_{j} \geq \tilde{R}_{0}(\omega)\right) \geq \eta\right\} \geq \lambda \\
\Leftrightarrow \sum_{j=1}^{n}\left(R_{j}^{1}+T^{*}(1-\lambda) R_{j}^{2}\right) x_{j}+R^{*}(\eta) \sum_{j=1}^{n} \gamma_{j} x_{j} \geq R_{0}^{0}+T^{*}(1-\lambda) R_{0}^{2}-\beta_{0} L^{*}(\eta)
\end{array}\right.
\end{aligned}
$$

where $T^{*}, L^{*}$ and $R^{*}$ are pseudo inverse functions defined as:

$T^{*}(\lambda)=\inf \{t \mid T(t) \geq \lambda\}, L^{*}(\lambda)=\sup \{t \mid L(t) \geq \lambda\}$ and $R^{*}(\lambda)=\sup \{t \mid R(t) \geq \lambda\}$.

Now the optimal solution of Problem 2 is equal to the following linear fractional programming problem:

\section{Problem 3}

$$
\begin{aligned}
& \max \quad \sum_{j=1}^{n}\left(R_{j}^{1}+T^{*}(1-\lambda) R_{j}^{2}\right) x_{j}+R^{*}(\eta) \sum_{j=1}^{n} \gamma_{j} x_{j} \\
& \text { subject to } \quad \sum_{j=1}^{n} x_{j}=M_{0}, \\
& \quad \sum_{j=1}^{n}\left(R_{j}^{1}+T^{*}(1-\lambda) R_{j}^{2}\right) x_{j}+R^{*}(\eta) \sum_{j=1}^{n} \gamma_{j} x_{j} \geq R_{0}^{0}+T^{*}(1-\lambda) R_{0}^{2}-\beta_{0} L^{*}(\eta), \\
& \quad 0 \leq x_{j} \leq U_{j} ; \quad j=1,2, \ldots, n .
\end{aligned}
$$

This can be solved by one of the LP solver (such as LINGO) and obtained an optimal solution for portfolio selection problem.

\subsection{Necessity-based model}

The possibility-based model may be improper since the obtain solution will be too optimistic, so necessity-based model can be suitable for pessimistic decision maker who wishes to avoid risk.

The degree of necessity $N(\tilde{Z}(\omega) \geq f)$ for fuzzy constraint $(\tilde{Z}(\omega) \geq f)$ under the possibility distribution $\mu_{\tilde{Z}(\omega)}(t)$ is defined as follows:

$$
N(\tilde{Z}(\omega) \geq f)=\inf _{y_{1}, y_{2}}\left\{\max \left\{1-\mu_{\tilde{Z}(\omega)}\left(y_{1}\right), 1-\mu_{f}\left(y_{2}\right)\right\} \mid y_{1} \geq y_{2}\right\} \geq \eta
$$

The necessity degree of fuzzy constraint $\left(\sum_{j=1}^{n} \tilde{R}_{j}(\omega) x_{j} \geq \tilde{R}_{0}(\omega)\right)$ under the possibility distributions is defined as follows:

$$
N\left(\sum_{j=1}^{n} \tilde{R}_{j}(\omega) x_{j} \geq \tilde{R}_{0}(\omega)\right)=\inf _{y_{1}, y_{2}}\left\{\max \left\{1-\mu_{\substack{\sum_{j=1} \tilde{R}_{j}(\omega) x_{j} \\ j(1)}}\left(y_{1}\right), 1-\mu_{\tilde{R}_{0}(\omega)}\left(y_{2}\right)\right\} \mid y_{1} \geq y_{2}\right\}
$$

We maximize the degree of necessity $N(\tilde{Z}(\omega) \geq f)$ and the degree of necessity $\left(\sum_{j=1}^{n} \tilde{R}_{j}(\omega) x_{j} \geq \tilde{R}_{0}(\omega)\right)$, therefore our portfolio selection model in problem 1 come by following model: 


\section{Problem 4}

$\max f$

subject to $\operatorname{Pr}\{\omega \mid N(\tilde{Z}(\omega) \geq f) \geq \eta\} \geq \lambda$,

$$
\begin{aligned}
& \sum_{j=1}^{n} x_{j}=M_{0}, \\
& \operatorname{Pr}\left\{\omega \mid N\left(\sum_{j=1}^{n} \tilde{R}_{j}(\omega) x_{j} \geq \tilde{R}_{0}(\omega)\right) \geq \eta\right\} \geq \lambda, \\
& 0 \leq x_{j} \leq U_{j} ; \quad j=1,2, \ldots, n .
\end{aligned}
$$

where $\lambda$ is a predetermined probability level and $\eta$ is a predetermined possibility level. A feasible solution of portfolio selection problem is called a necessity solution. In order to transform the above model to a linear programming model, we need to reformulate Eq. (23) and Eq. (25). Consider the following theorem:

Theorem 2: (Katagiri et al. 2008)

Let ${ }_{{ }_{j}}$ be a positive decision vector then:

$$
\left\{\begin{array}{l}
\text { 1) } \operatorname{Pr}\{\omega \mid N(\tilde{Z}(\omega) \geq f) \geq \eta\} \geq \lambda \\
\Leftrightarrow \sum_{j=1}^{n}\left(R_{j}^{0}+T^{*}(1-\lambda) R_{j}^{2}\right) x_{j}-L^{*}(1-\eta) \sum_{j=1}^{n} \beta_{j} x_{j} \geq f \\
\text { 2) } \operatorname{Pr}\left\{\omega \mid N\left(\sum_{j=1}^{n} \tilde{R}_{j}(\omega) x_{j} \geq \tilde{R}_{0}(\omega)\right) \geq \eta\right\} \geq \lambda \\
\Leftrightarrow \sum_{j=1}^{n}\left(R_{j}^{0}+T^{*}(1-\lambda) R_{j}^{2}\right) x_{j}-L^{*}(1-\eta) \sum_{j=1}^{n} \beta_{j} x_{j} \geq R_{0}^{0}+T^{*}(1-\lambda) R_{0}^{2}-\beta_{0} L^{*}(1-\eta)
\end{array}\right.
$$

where $T^{*}, L^{*}$ and $R^{*}$ are pseudo inverse functions defined as:

$$
T^{*}(\lambda)=\inf \{t \mid T(t) \geq \lambda\}, L^{*}(\lambda)=\sup \{t \mid L(t) \geq \lambda\} \text { and } R^{*}(\lambda)=\sup \{t \mid R(t) \geq \lambda\} \text {. }
$$

Now the optimal solution of Problem 4 is equal to the following linear parametric programming problem:

\section{Problem 5}

$$
\begin{aligned}
& \max \quad \sum_{j=1}^{n}\left(R_{j}^{0}+T^{*}(1-\lambda) R_{j}^{2}\right) x_{j}-L^{*}(1-\eta) \sum_{j=1}^{n} \beta_{j} x_{j} \\
& \text { subject to } \quad \sum_{j=1}^{n} x_{j}=M_{0}, \\
& \quad \sum_{j=1}^{n}\left(R_{j}^{0}+T^{*}(1-\lambda) R_{j}^{2}\right) x_{j}-L^{*}(1-\eta) \sum_{j=1}^{n} \beta_{j} x_{j} \geq R_{0}^{0}+T^{*}(1-\lambda) R_{0}^{2}-\beta_{0} L^{*}(1-\eta) \\
& \quad 0 \leq x_{j} \leq U_{j} ; \quad j=1,2, \ldots, n .
\end{aligned}
$$

This can be solved by one of the LP solver (such as LINGO) to determine the optimal solution of portfolio selection problem. Sadati and Nematian (2013) introduced fuzzy random portfolio problem as a two-level linear programming to calculate the upper bound and lower bound of the objective function value separately based on the decision maker opinion and according to the possibility and necessity-based model we reformulated the fuzzy random portfolio optimization to the linear programming. In this paper, our purpose is to find the optimum solution of portfolio optimization 
when we want to use possibility and necessity (optimistic and pessimistic approach) in one problem, so we have to reformulate our problem as a multiobjective problem and put both objective functions of problem 3 and 5 in one problem. Therefore, our multiobjective portfolio optimization formulates as:

\section{Problem 6}

$$
\begin{aligned}
& \max \sum_{j=1}^{n}\left(R_{j}^{1}+T^{*}(1-\lambda) R_{j}^{2}\right) x_{j}+R^{*}(\eta) \sum_{j=1}^{n} \gamma_{j} x_{j} \\
& \max \sum_{j=1}^{n}\left(R_{j}^{0}+T^{*}(1-\lambda) R_{j}^{2}\right) x_{j}-L^{*}(1-\eta) \sum_{j=1}^{n} \beta_{j} x_{j} \\
& \text { subject to } \quad \sum_{j=1}^{n} x_{j}=M_{0}, \\
& \sum_{j=1}^{n}\left(R_{j}^{1}+T^{*}(1-\lambda) R_{j}^{2}\right) x_{j}+R^{*}(\eta) \sum_{j=1}^{n} \gamma_{j} x_{j} \geq R_{0}^{0}+T^{*}(1-\lambda) R_{0}^{2}-\beta_{0} L^{*}(\eta), \\
& \sum_{j=1}^{n}\left(R_{j}^{0}+T^{*}(1-\lambda) R_{j}^{2}\right) x_{j}-L^{*}(1-\eta) \sum_{j=1}^{n} \beta_{j} x_{j} \geq R_{0}^{0}+T^{*}(1-\lambda) R_{0}^{2}-\beta_{0} L^{*}(1-\eta) \\
& \quad 0 \leq x_{j} \leq U_{j} ; \quad j=1,2, \ldots, n .
\end{aligned}
$$

To solve this multiobjective problem we use the concepts of optimistic and pessimistic. As we know that possibility-based model may be improper since the obtain solution will be too optimistic, so necessity -based model can be suitable for optimistic decision maker who wishes to avoid risk. To find the optimum solution for this multiobjective portfolio optimization we present two ways: first we find the optimum solution just for single objective of possibility-based model with all constraints (possibility and necessity-based model constraints) after we find the solution, we use this optimum solution as necessity-based model's constraint. It means the objective function value for single objective possibility-based model becomes as a constraint for necessity-based model in the next step and finally we find the optimum solution for single objective of necessity -based model. This way completely is illustrated by following model:

First, we will find the optimum solution for single objective of possibility-base model without computing the objective function of necessity-based model:

\section{Problem 7}

$$
\begin{aligned}
& \max \sum_{j=1}^{n}\left(R_{j}^{1}+T^{*}(1-\lambda) R_{j}^{2}\right) x_{j}+R^{*}(\eta) \sum_{j=1}^{n} \gamma_{j} x_{j} \\
& \text { subject to } \quad \sum_{j=1}^{n} x_{j}=M_{0}, \\
& \quad \sum_{j=1}^{n}\left(R_{j}^{1}+T^{*}(1-\lambda) R_{j}^{2}\right) x_{j}+R^{*}(\eta) \sum_{j=1}^{n} \gamma_{j} x_{j} \geq R_{0}^{0}+T^{*}(1-\lambda) R_{0}^{2}-\beta_{0} L^{*}(\eta), \\
& \quad \sum_{j=1}^{n}\left(R_{j}^{0}+T^{*}(1-\lambda) R_{j}^{2}\right) x_{j}-L^{*}(1-\eta) \sum_{j=1}^{n} \beta_{j} x_{j} \geq R_{0}^{0}+T^{*}(1-\lambda) R_{0}^{2}-\beta_{0} L^{*}(1-\eta) \\
& \quad 0 \leq x_{j} \leq U_{j} ; \quad j=1,2, \ldots, n .
\end{aligned}
$$


After solving this problem by Lingo we find the objective function value(OFV) of problem 7 then with this result we determine the necessity-based model with extra constraint stated in Eq. (37) resulted according to problem 7, Therefore, we have:

\section{Problem 8}

$$
\begin{array}{ll}
\max & \sum_{j=1}^{n}\left(R_{j}^{0}+T^{*}(1-\lambda) R_{j}^{2}\right) x_{j}-L^{*}(1-\eta) \sum_{j=1}^{n} \beta_{j} x_{j} \\
\text { subject to } & \sum_{j=1}^{n}\left(R_{j}^{1}+T^{*}(1-\lambda) R_{j}^{2}\right) x_{j}+R^{*}(\eta) \sum_{j=1}^{n} \gamma_{j} x_{j}=\text { OFV(problem 7) } \\
& \sum_{j=1}^{n} x_{j}=M_{0}, \\
& \sum_{j=1}^{n}\left(R_{j}^{1}+T^{*}(1-\lambda) R_{j}^{2}\right) x_{j}+R^{*}(\eta) \sum_{j=1}^{n} \gamma_{j} x_{j} \geq R_{0}^{0}+T^{*}(1-\lambda) R_{0}^{2}-\beta_{0} L^{*}(\eta), \\
& \sum_{j=1}^{n}\left(R_{j}^{0}+T^{*}(1-\lambda) R_{j}^{2}\right) x_{j}-L^{*}(1-\eta) \sum_{j=1}^{n} \beta_{j} x_{j} \geq R_{0}^{0}+T^{*}(1-\lambda) R_{0}^{2}-\beta_{0} L^{*}(1-\eta) \\
& 0 \leq x_{j} \leq U_{j} ; \quad j=1,2, \ldots, n .
\end{array}
$$

By solving this problem we find the optimum solution of multiobjective portfolio optimization. The second way to find the optimum solution for this multiobjective portfolio optimization is same as first way but at first, we find the single objective of necessity-based model and after the finding of optimum solution of necessity-based model we use this result as a constraint for possibility -based model and finally same as above we will find the optimum solution for possibility-based model.

Therefore, we have:

Problem 9

$$
\begin{aligned}
& \max \quad \sum_{j=1}^{n}\left(R_{j}^{0}+T^{*}(1-\lambda) R_{j}^{2}\right) x_{j}-L^{*}(1-\eta) \sum_{j=1}^{n} \beta_{j} x_{j} \\
& \text { subject to } \quad \sum_{j=1}^{n} x_{j}=M_{0}, \\
& \qquad \sum_{j=1}^{n}\left(R_{j}^{1}+T^{*}(1-\lambda) R_{j}^{2}\right) x_{j}+R^{*}(\eta) \sum_{j=1}^{n} \gamma_{j} x_{j} \geq R_{0}^{0}+T^{*}(1-\lambda) R_{0}^{2}-\beta_{0} L^{*}(\eta), \\
& \sum_{j=1}^{n}\left(R_{j}^{0}+T^{*}(1-\lambda) R_{j}^{2}\right) x_{j}-L^{*}(1-\eta) \sum_{j=1}^{n} \beta_{j} x_{j} \geq R_{0}^{0}+T^{*}(1-\lambda) R_{0}^{2}-\beta_{0} L^{*}(1-\eta) \\
& \quad 0 \leq x_{j} \leq U_{j} ; \quad j=1,2, \ldots, n .
\end{aligned}
$$

Similar to problem 7, we find the objective function value(OFV) then with this result we determine the possibility-based model with extra constraint (48) which has been resulted according problem 10, Therefore, we have: 
Problem 10

$$
\begin{array}{ll}
\max & \sum_{j=1}^{n}\left(R_{j}^{1}+T^{*}(1-\lambda) R_{j}^{2}\right) x_{j}+R^{*}(\eta) \sum_{j=1}^{n} \gamma_{j} x_{j} \\
\text { subject to } \quad \sum_{j=1}^{n}\left(R_{j}^{0}+T^{*}(1-\lambda) R_{j}^{2}\right) x_{j}-L^{*}(1-\eta) \sum_{j=1}^{n} \beta_{j} x_{j}=\text { OFV(problem 9) } \\
\\
\sum_{j=1}^{n} x_{j}=M_{0}, \\
\sum_{j=1}^{n}\left(R_{j}^{1}+T^{*}(1-\lambda) R_{j}^{2}\right) x_{j}+R^{*}(\eta) \sum_{j=1}^{n} \gamma_{j} x_{j} \geq R_{0}^{0}+T^{*}(1-\lambda) R_{0}^{2}-\beta_{0} L^{*}(\eta), \\
\sum_{j=1}^{n}\left(R_{j}^{0}+T^{*}(1-\lambda) R_{j}^{2}\right) x_{j}-L^{*}(1-\eta) \sum_{j=1}^{n} \beta_{j} x_{j} \geq R_{0}^{0}+T^{*}(1-\lambda) R_{0}^{2}-\beta_{0} L^{*}(1-\eta) \\
0 \leq x_{j} \leq U_{j} ; \quad j=1,2, \ldots, n .
\end{array}
$$

By solving this problem, we obtain the second type of optimum solution for our portfolio selection. In next section with a numerical example, we will illustrate whole aspects of our approach.

\section{Numerical example}

In this section, an example is given to illustrate the proposed possibility and necessity-based model for portfolio optimization selection. We believe that an investment plan needs to consider not only the historical data, but also new information. Therefore, we decided to use the second type of data, which have been received after starting the first decision. Let us consider 5 securities whose returns are fuzzy random variables and their values are given in Table 1. $\bar{t}$ is a normal random variable whose mean 0 and variance 1 . The upper bound of investment amount in each stock is set to no more than 60 units of the total available fund. Given a total allocation budget of 200 units and annual return which is fuzzy random variable is shown as $\tilde{\bar{R}}=M_{0} \tilde{\bar{r}_{0}}$ where $\tilde{\overline{r_{0}}}=(1+0.3 \bar{t}, 1+0.3 \bar{t}, 0.3,0.3)$.

Now we want to know what is the optimal solution for our portfolio selection problem for the different levels of probability and possibility $\{0.1,0.4,0.7,0.9\}$.

We apply the possibility and necessity-based model based on theorems 1and 2 to obtained fuzzy random portfolio selection problem. First of all we calculate possibility and necessity-based model separately, and then calculate the multiobjective portfolio optimizations according to the two ways have been presented. All the results are collected in tables 2 to 5.

Table 1

Parameters of the example

\begin{tabular}{ccccccc}
\hline$j$ & 0 & 1 & 2 & 3 & 4 & 5 \\
\hline$R_{j}^{0}$ & 200 & 1.2 & 1.25 & 1.35 & 1.25 & 1.4 \\
$R_{j}^{1}$ & 200 & 1.35 & 1.3 & 1.45 & 1.35 & 1.5 \\
$R_{j}^{2}$ & 60 & 0.5 & 0.6 & 0.55 & 0.4 & 0.5 \\
$\beta_{j}$ & 60 & 0.15 & 0.1 & 0.2 & 0.15 & 0.2 \\
$\gamma_{j}$ & 60 & 0.15 & 0.1 & 0.2 & 0.15 & 0.2 \\
\hline
\end{tabular}


Table 2

Numerical results (possibility-based model)

\begin{tabular}{ccccc}
\hline$\lambda, \eta$ & 0.1 & 0.4 & 0.7 & 0.9 \\
\hline$x_{1}^{*}$ & 20 & 0 & 0 & 20 \\
$x_{2}^{*}$ & 60 & 60 & 20 & 0 \\
$x_{3}^{*}$ & 60 & 60 & 60 & 60 \\
$x_{4}^{*}$ & 0 & 20 & 60 & 60 \\
$x_{5}^{*}$ & 60 & 60 & 60 & 60 \\
\hline OFV $^{\mathrm{a}}$ & 451.22 & 331.85 & 244.39 & 164.44 \\
\hline
\end{tabular}

Table 3

Numerical results (necessity-based model)

\begin{tabular}{|c|c|c|c|c|}
\hline$\lambda, \eta$ & 0.1 & 0.4 & 0.7 & 0.9 \\
\hline$x_{1}^{*}$ & 20 & 0 & 0 & 20 \\
\hline$x_{2}^{*}$ & 60 & 60 & 20 & 0 \\
\hline$x_{3}^{*}$ & 60 & 60 & 60 & 60 \\
\hline$X_{4}^{*}$ & 0 & 20 & 60 & 60 \\
\hline$x_{5}^{*}$ & 60 & 60 & 60 & 60 \\
\hline $\mathrm{OFV}^{\mathrm{a}}$ & 400.22 & 278.55 & 188.03 & 107.44 \\
\hline
\end{tabular}

${ }^{\mathrm{a}}$ Objective function value.

Table 4

Numerical results (multiobjective portfolio optimization according to first way)

\begin{tabular}{ccccc}
\hline$\lambda, \eta$ & 0.1 & 0.4 & 0.7 & 0.9 \\
\hline$x_{1}^{*}$ & 20 & 60 & 20 & 20 \\
$x_{2}^{*}$ & 60 & 0 & 0 & 0 \\
$x_{3}^{*}$ & 60 & 60 & 60 & 60 \\
$x_{4}^{*}$ & 0 & 20 & 60 & 60 \\
$x_{5}^{*}$ & 60 & 60 & 60 & 60 \\
\hline $\mathrm{OFV}^{\mathrm{b}}$ & 451.22 & 332.39 & 244.39 & 164.44 \\
$\mathrm{OFV}^{\mathrm{b}}$ & 400.22 & 272.55 & 187.39 & 107.44 \\
\hline
\end{tabular}

${ }^{\mathrm{b}}$ Objective function value of Possibility and Necessity-based model

Table 5

Numerical results (multiobjective portfolio optimization according to second way)

\begin{tabular}{ccccc}
\hline$\lambda, \eta$ & 0.1 & 0.4 & 0.7 & 0.9 \\
\hline$x_{1}^{*}$ & 20 & 0 & 0 & 20 \\
$x_{2}^{*}$ & 60 & 60 & 20 & 0 \\
$x_{3}^{*}$ & 60 & 60 & 60 & 60 \\
$x_{4}^{*}$ & 0 & 20 & 60 & 60 \\
$X_{5}^{*}$ & 60 & 60 & 60 & 60 \\
\hline OFV $^{\mathrm{b}}{ }_{\mathrm{P}}$ & 451.22 & 328.55 & 242.03 & 164.44 \\
OFV $_{\mathrm{N}}$ & 400.22 & 278.55 & 188.03 & 107.44 \\
\hline
\end{tabular}


Clearly, the greater the $\lambda, \eta$ value, the greater the level of possibility and the lower the objective function value is. The numerical results of Table 2 and Table 3 have been calculated separately but results of Table 4 and Table 5 are based on the multiobjective problem, which show how possibility and necessity approach influence each other and these results are helpful for decision makers who are in ambiguity to choose which approach to find their solution in portfolio selection. In other words, when they use only possibility-based model for their portfolio selection, after receiving their optimum solution, they found their solution too optimistic and then try to use pessimistic approach of necessity-based model and it keeps them in ambiguity to choose which approach. Therefore, for these kinds of decision makers, the best approach is to use multiobjective portfolio selection, which has been indicated in problem 6 . The comparisons between single objective and multiobjective are depicted in Fig 1 and Fig 2.

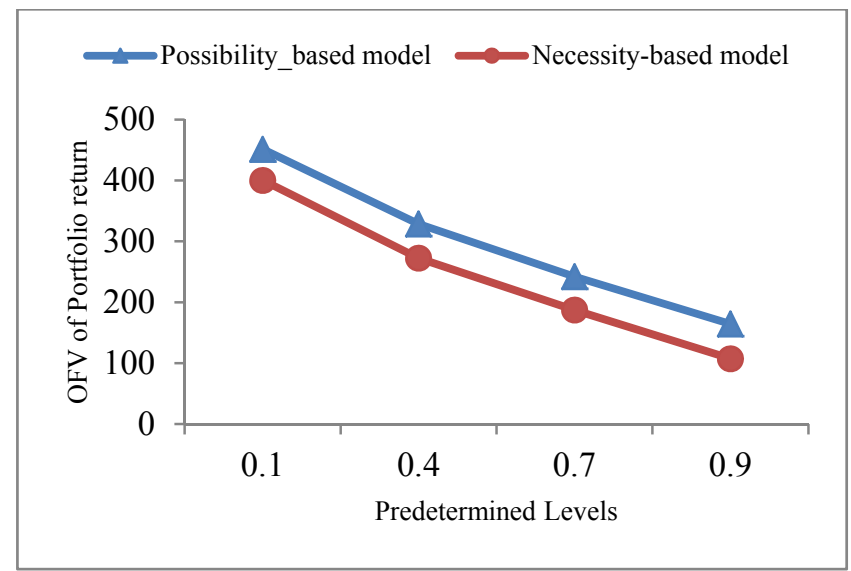

Fig. 1. Comparison of Possibility and Necessity-based model in single objective

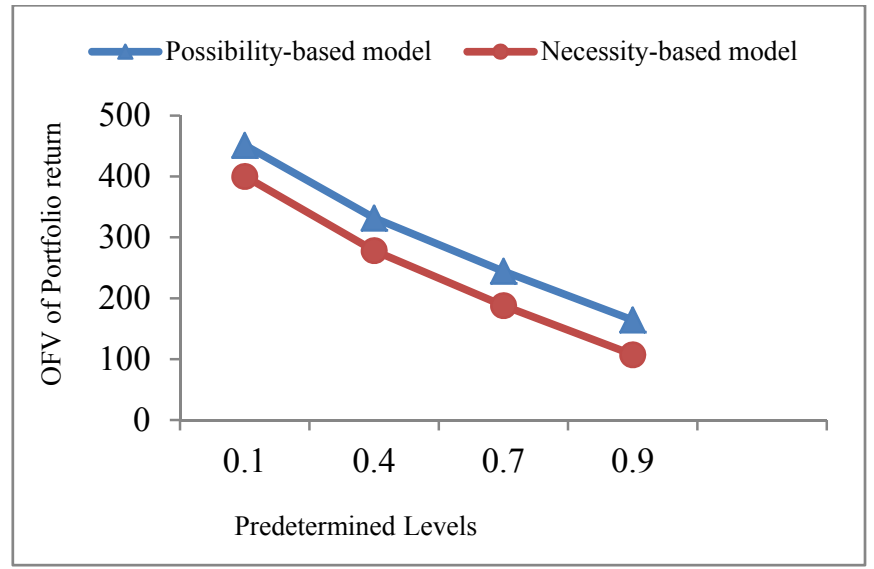

Fig. 2. Comparison of Possibility and Necessity-based model in multiobjective

\section{Conclusion}

Portfolio optimization has been one of the most important fields of research in economic and finance. Since the prospective returns of assets used for portfolio optimization problem are forecasted values, considerable uncertainty is involved. In this paper, Markowitz's mean-variance idea was extended to portfolio selection by possibility and necessity-based model. This paper proposed a solution method for portfolio selection model whose parameters were fuzzy random variables. The idea was based on possibility and necessity-based model. We first presented the possibility and necessity-based model to reformulate the fuzzy random portfolio selection model to linear programming, then with these two kind of linear programs we constructed the multiobjective problem. The optimum solution of multiobjective problem based on the illustrated example was helpful for decision makers who are in ambiguity to choose which approach to find their solution in portfolio selection (optimistic or pessimistic approach). For future research, we will apply the other methods for fuzzy random portfolio selection model and improve our portfolio selection problem.

\section{References}

Arenas Parra, M., Bilbao Terol, A., \& Rodriguez Uria, M. V. (2001). A fuzzy goal programming approach to portfolio selection. European Journal of Operational Research, 133(2), 287-297.

Black, F., \& Litterman, R. B. (1991). Asset allocation: combining investor views with market equilibrium. The Journal of Fixed Income, 1(2), 7-18.

Chow, K. V., \& Denning, K. C. (1994). On variance and lower partial moment betas the equivalence of systematic risk measures. Journal of Business Finance \& Accounting, 21(2), 231-241.

Crama, Y., \& Schyns, M. (2003). Simulated annealing for complex portfolio selection problems. European Journal of operational research, 150(3), 546-571. 
Dastkhan, H., Golmakani, H. R., \& Gharneh, N. S. (2013). How to obtain a series of satisfying portfolios: a fuzzy portfolio management approach.International Journal of Industrial and Systems Engineering, 14(3), 333-351.

Gharakhani, M., \& Sadjadi, S. (2013). A fuzzy compromise programming approach for the BlackLitterman portfolio selection model. Decision Science Letters, 2(1), 11-22.

Gil, M. Á., López-Díaz, M., \& Ralescu, D. A. (2006). Overview on the development of fuzzy random variables. Fuzzy sets and systems, 157(19), 2546-2557.

Grootveld, H., \& Hallerbach, W. (1999). Variance vs downside risk: Is there really that much difference?. European Journal of operational research, 114(2), 304-319.

Hao, F. F., \& Liu, Y. K. (2008). Portfolio Selection Problem in Fuzzy Random Decision Systems. In Innovative Computing Information and Control, 2008. ICICIC'08. 3rd International Conference on (pp. 271-271). IEEE.

Hao, F. F., \& Liu, Y. K. (2009). Mean-variance models for portfolio selection with fuzzy random returns. Journal of Applied Mathematics and Computing,30(1-2), 9-38.

Harlow, W. V., \& Rao, R. K. (1989). Asset pricing in a generalized mean-lower partial moment framework: Theory and evidence. Journal of Financial and Quantitative Analysis, 24(3), 285-311.

Katagiri, H., Sakawa, M., Kato, K., \& Nishizaki, I. (2008). Interactive multiobjective fuzzy random linear programming: Maximization of possibility and probability. European Journal of Operational Research, 188(2), 530-539.

Konno, H., \& Yamazaki, H. (1991). Mean-absolute deviation portfolio optimization model and its applications to Tokyo stock market. Management science, 37(5), 519-531.

Kwakernaak Kwakernaak, H. (1978). Fuzzy random variables-I. Definitions and theorems. Information Sciences, 15(1), 1-29.

Li, J., \& Xu, J. (2009). A novel portfolio selection model in a hybrid uncertain environment. Omega, 37(2), 439-449.

Liu, B. (2009). Theory and practice of uncertain programming (Vol. 239). Springer.

Liu, Y. J., Zhang, W. G., \& Xu, W. J. (2012). Fuzzy multi-period portfolio selection optimization models using multiple criteria. Automatica, 48(12), 3042-3053.

Markowitz, H. (1952). Portfolio selection*. The journal of finance, 7(1), 77-91.

Markowitz, H. (1959). Portfolio selection: efficient diversification of investments (No. 16). Yale university press.

Markowitz, H., Todd, P., Xu, G., \& Yamane, Y. (1993). Computation of mean-semivariance efficient sets by the critical line algorithm. Annals of Operations Research, 45(1), 307-317.

Plat, R. (2009). Stochastic portfolio specific mortality and the quantification of mortality basis risk. Insurance: Mathematics and Economics, 45(1), 123-132.

Puri, M. L., \& Ralescu, D. A. (1986). Fuzzy random variables. Journal of mathematical analysis and applications, 114(2), 409-422.

Sadati, M. E. H., \& Nematian, J. (2013). Two-level linear programming for fuzzy random portfolio optimization through possibility and necessity-based model.

Sadjadi, S. J., Seyedhosseini, S. M., \& Hassanlou, K. (2011). Fuzzy multi period portfolio selection with different rates for borrowing and lending. Applied Soft Computing, 11(4), 3821-3826.

Sadjadi, S. J., Gharakhani, M., \& Safari, E. (2012). Robust optimization framework for cardinality constrained portfolio problem. Applied Soft Computing,12(1), 91-99.

Shapiro, A. F. (2009). Fuzzy random variables. Insurance: Mathematics and Economics, 44(2), 307314.

Sakawa, M. (1993). Fuzzy sets and interactive multiobjective optimization. New York: Plenum.

Woodside-Oriakhi, M., Lucas, C., \& Beasley, J. E. (2013). Portfolio rebalancing with an investment horizon and transaction costs. Omega, 41(2), 406-420.

Xia, Y., Liu, B., Wang, S., \& Lai, K. K. (2000). A model for portfolio selection with order of expected returns. Computers \& Operations Research, 27(5), 409-422.

Yu, J. R., \& Lee, W. Y. (2011). Portfolio rebalancing model using multiple criteria. European Journal of Operational Research, 2009(2), 166-175. 
Zadeh, L. A. (1965). Fuzzy sets. Information and control, 8(3), 338-353.

Zhang, W. G., Liu, Y. J., \& Xu, W. J. (2013). A new fuzzy programming approach for multi-period portfolio optimization with return demand and risk control. Fuzzy Sets and Systems. 